\section{PPRA/PCMSO: auditoria, inspeção do trabalho e controle social}

\author{
Environment risk prevention programs and \\ occupational health monitoring programs: audits, \\ labor inspection, and social control
}

Carlos Roberto Miranda 1

Carlos Roberto Dias 1

\footnotetext{
1 Delegacia Regional do Trabalho na Bahia Ministério do Trabalho e Emprego, Salvador, Brasil.

Correspondência Carlos Roberto Miranda Delegacia Regional do Trabalho na Bahia, Ministério do Trabalho e Emprego. Rua Magno Valente 68/1001,Salvador, BA

41810-620, Brasil.

miranda@saudeetrabalho.com.br
}

\section{Abstract}

The authors analyzed environmental risk prevention programs and occupational health monitoring programs implemented by $30 \mathrm{com}$ panies from various industrial sectors, each with more than 100 employees, in Salvador, Bahia, Brazil. Observed inconsistencies were studied according to occupational risk, considering the different stages of development in the programs. The study suggested low technical quality in these programs, revealing the need to increase the range of government inspection and to stimulate worker and trade union participation in such programs. The development and improvement of inspection procedures, instruments, and protocols were also considered fundamentally important for health and safety in the workplace.

Environmental Risks; Occupational Health; Occupational Risk

\section{Introdução}

A legislação brasileira que trata da segurança e da saúde no trabalho passou a adotar um novo enfoque, a partir do final de 1994, ao estabelecer a obrigatoriedade das empresas elaborarem e implementarem dois programas: um ambiental, o Programa de Prevenção de Riscos Ambientais (PPRA), e outro médico, o Programa de Controle Médico de Saúde Ocupacional (PCMSO). Ad otando como paradigma a Convenção 161/85 da Organização Internacional do Trabalho (OIT), a legislação brasileira específica passou a considerar as questões incidentes não somente sobre o indivíduo mas também sobre a coletividade de trabalhadores, promovendo, assim, uma ampliação do conceito restrito de "medicina do trabalho" 1,2 .

Em verdade, apesar de o Brasil ter ratificado em 1991 a Convenção 161 da OIT, até 1994 as Normas Regulamentadoras (NR) caracterizavam-se ainda por um enfoque essencialmente "individualista". As NR-7 e 9 intitulavam-se, respectivamente, Exames Médicos e Riscos Ambientais, ou seja, a ênfase era, isoladamente, ora para o corpo do trabalhador, ora para a avaliação quantitativa de um certo risco ambiental. As novas normas, preocupadas agora com a saúde do conjunto dos trabalhadores, privilegiaram o instrumental clínicoepidemiológico na abordagem da relação saúde/trabalho e introduziram a questão da valo- 
rização da participação dos trabalhadores e do controle social. Neste sentido, a exigência legal dos novos programas PCMSO e PPRA representou, na prática, a superação de um "viés biologista/ambiental" e a introdução de um "olhar coletivo" nas questões relacionadas com a segurança e a saúde dos trabalhadores brasileiros 3,4 .

Programa de Prevenção

de Riscos Ambientais

O PPRA, cuja obrigatoriedade foi estabelecida pela NR-9 da Portaria 3.214/78, apesar de seu caráter multidisciplinar, é considerado essencialmente um programa de higiene ocupacional que deve ser implementado nas empresas de forma articulada com um programa médico - o PCMSO 5,6.

Todas as empresas, independente do número de empregados ou do grau de risco de suas atividades, estão obrigadas a elaborar e implementar o PPRA, que tem como objetivo a prevenção e o controle da exposição ocupacional aos riscos ambientais, isto é, a prevenção e o controle dos riscos químicos, físicos e biológicos presentes nos locais de trabalho. A NR-9 detalha as etapas a serem cumpridas no desenvolvimento do programa, os itens que compõem a etapa do reconhecimento dos riscos, os limites de tolerância adotados na etapa de avaliação e os conceitos que envolvem as medidas de controle. A norma estabelece, ainda, a obrigatoriedade da existência de um cronograma que indique claramente os prazos para o desenvolvimento das diversas etapas e para o cumprimento das metas estabelecidas.

Um aspecto importante deste programa é que ele pode ser elaborado dentro dos conceitos mais modernos de gerenciamento e gestão, em que o empregador tem autonomia suficiente para, com responsabilidade, adotar um conjunto de medidas e ações que considere necessárias para garantir a saúde e a integridade física dos seus trabalhadores. A elaboração, implementação e avaliação do PPRA podem ser feitas por qualquer pessoa, ou equipe de pessoas que, a critério do empregador, sejam capazes de desenvolver o disposto na norma. Além disso, cabe à própria empresa estabelecer as estratégias e as metodologias que serão utilizadas para o desenvolvimento das ações, bem como a forma de registro, manutenção e divulgação dos dados gerados no desenvolvimento do programa.
As ações do PPRA devem ser desenvolvidas no âmbito de cada estabelecimento da empresa, e sua abrangência e profundidade dependem das características dos riscos existentes no local de trabalho e das respectivas necessidades de controle.

A NR-9 estabelece as diretrizes gerais e os parâmetros mínimos a serem observados na execução do programa; porém, os mesmos podem ser ampliados mediante negociação coletiva de trabalho. Procurando garantir a efetiva implementação do PPRA, a norma estabelece que a empresa deve adotar mecanismos de avaliação que permitam verificar o cumprimento das etapas, das ações e das metas previstas. Além disso, a NR-9 prevê algum tipo de controle social, garantindo aos trabalhadores o direito à informação e à participação no planejamento e no acompanhamento da execução do programa.

\section{Programa de Controle Médico de Saúde Ocupacional}

O PCMSO, cuja obrigatoriedade foi estabelecida pela NR-7 da Portaria 3.214/78, é um programa médico que deve ter caráter de prevenção, rastreamento e diagnóstico precoce dos agravos à saúde relacionados ao trabalho. Entendese aqui por "diagnóstico precoce", segundo o conceito adotado pela Organização Mundial da Saúde (OMS), a detecção de distúrbios dos mecanismos compensatórios e homeostáticos, enquanto ainda permanecem reversíveis alterações bioquímicas, morfológicas e funcionais.

Todas as empresas, independente do número de empregados ou do grau de risco de sua atividade, estão obrigadas a elaborar e implementar o PCMSO, que deve ser planejado e implantado com base nos riscos à saúde dos trabalhadores, especialmente os riscos identificados nas avaliações previstas no PPRA. Entre suas diretrizes, uma das mais importantes é aquela que estabelece que o PCMSO deve considerar as questões incidentes tanto sobre o indivíduo como sobre a coletividade de trabalhadores, privilegiando o instrumental clínicoepidemiológico. A norma estabelece, ainda, o prazo e a periodicidade para a realização das avaliações clínicas, assim como define os critérios para a execução e interpretação dos exames médicos complementares (os indicadores biológicos).

Em síntese, na elaboração do PCMSO, o mínimo requerido é um estudo prévio para re- 
conhecimento dos riscos ocupacionais existentes na empresa, por intermédio de visitas aos locais de trabalho, baseando-se nas informações contidas no PPRA. Com base neste reconhecimento de riscos, deve ser estabelecido um conjunto de exames clínicos e complementares específicos para cada grupo de trabalhadores da empresa, utilizando-se de conhecimentos científicos atualizados e em conformidade com a boa prática médica. Logo, o nível de complexidade do PCMSO depende basicamente dos riscos existentes em cada empresa, das exigências físicas e psíquicas das atividades desenvolvidas e das características biopsicofisiológicas de cada população trabalhadora 7. A norma estabelece as diretrizes gerais e os parâmetros mínimos a serem observados na execução do programa, podendo os mesmos, entretanto, ser ampliados pela negociação coletiva de trabalho.

O PCMSO deve ser coordenado por um médico, com especialização em medicina do trabalho, que será o responsável pela execução do programa. Ao empregador, por sua vez, compete garantir a elaboração e efetiva implementação do PCMSO, tanto quanto zelar pela sua eficácia. Procurando garantir a efetiva implementação do PCMSO, a NR-7 determina que o programa deverá obedecer a um planejamento em que estejam previstas as ações de saúde a serem executadas durante o ano, devendo estas ser objeto de relatório anual. O relatório anual deverá discriminar, por setores da empresa, o número e a natureza dos exames médicos, incluindo avaliações clínicas e exames complementares, estatísticas de resultados considerados anormais, assim como o planejamento para o ano seguinte.

\section{A inspeção do trabalho e os programas PPRA/PCMSO}

A inseparabilidade entre o trabalho e o indivíduo que o realiza, a implicação da pessoa do trabalhador na atividade laboral, determinam uma exigência de tutela de sua liberdade e integridade física, ou seja, em última instância determinam a intervenção do Estado na regulamentação das relações de trabalho. Em conseqüência, na medida em que o trabalho é de alguma forma normatizado, a inspeção encontra sentido e lugar de ser na história do trabalho. Em síntese, o serviço de inspeção deveria ser a forma de tornar efetivas as regulamentações do processo de trabalho 8 .

Contudo, não são poucas as dificuldades relacionadas à inspeção e ao controle dos ambientes de trabalho. A complexidade cada vez maior das relações trabalhistas exige que o Auditor do Trabalho tenha uma boa formação jurídica e técnica. O caráter multidisciplinar da inspeção do trabalho justifica a incorporação de carreiras técnicas que aportem ao sistema de inspeção e proteção do trabalho os conhecimentos teóricos e práticos que são necessários para atender adequadamente as questões que se relacionam com a segurança e saúde dos trabalhadores 4 .

Neste sentido, a Convenção no 81 da OIT, adotada em 1947 e ratificada pelo Brasil em 1957, estabelece em seu artigo 10 que o número de inspetores de trabalho deve ser o suficiente para permitir o exercício eficaz das funções de serviço de inspeção e será fixado tendo-se em conta o número, a natureza, a importância e a situação dos estabelecimentos sujeitos ao controle da inspeção, assim como o número e a diversidade das categorias de trabalhadores ocupados nesses estabelecimentos 9 .

Em nosso país, o Ministério do Trabalho e Emprego (MTE) é o órgão de âmbito nacional competente para coordenar, orientar, controlar e supervisionar as atividades relacionadas com a segurança e saúde no trabalho, inclusive a fiscalização do cumprimento dos preceitos legais e regulamentares, em todo o Território Nacional. No plano estadual, essa fiscalização é executada pelas Delegacias Regionais do Trabalho. Para desenvolver a fiscalização na área de segurança e saúde no trabalho em todo o país, o MTE dispõe atualmente de 690 auditores fiscais. Vale observar que esse contingente é claramente insuficiente para inspecionar um total de mais de 4 milhões de estabelecimentos em atividade e dar cobertura a uma população economicamente ativa hoje em torno de 28 milhões de trabalhadores. Esses dados revelam a existência de um auditor fiscal para cerca de 6 mil estabelecimentos, ou seja, um auditor fiscal para cada 40 mil trabalhadores em atividade no país.

No caso do Estado da Bahia, o quadro é ainda mais grave, pois a Delegacia Regional do Trabalho dispõe de apenas vinte auditores na área de segurança e saúde no trabalho (11 médicos do trabalho e 9 engenheiros de segurança) para inspecionar cerca de 200 mil estabelecimentos em atividade no Estado e dar cobertura para uma população economicamente ativa hoje em torno de 1.300.000 trabalhadores. Ou seja, um auditor fiscal para cerca de 10 mil estabelecimentos, ou, um auditor fiscal para cada 65 mil trabalhadores em atividade 10,11.

Em relação ao PPRA, do ponto de vista da inspeção do trabalho, certos procedimentos obrigatórios previstos na NR-9 podem permitir 
um melhor acompanhamento do programa. Um aspecto fundamental é a obrigatoriedade do empregador reconhecer os riscos ambientais presentes nos diversos locais de trabalho da empresa e assumir prazos para solucionar as questões relativas a esses riscos. Como o programa é permanente, cabe ao empregador formalizar um cronograma anual, com estabelecimento das ações a serem executadas e as metas a serem alcançadas neste período. Por sua vez, a exigência da manutenção de um histórico com o registro dos dados mantido por um período mínimo de vinte anos permite aos $\mathrm{Au}$ ditores Fiscais do Trabalho verificar e comprovar tecnicamente os resultados alcançados no desenvolvimento do programa.

Quanto ao PCMSO, ao estabelecer a obrigatoriedade de um planejamento em que estejam previstas as ações de saúde a serem executadas durante o ano, que devem ser objeto de um relatório anual, a NR-7 possibilitou também um melhor acompanhamento do programa médico da empresa.

\section{Metodologia}

O presente trabalho teve como objetivo principal auditorar, do ponto de vista da inspeção do trabalho, PPRA e PCMSO elaborados e implementados por empresas, de diferentes ramos econômicos, com mais de cem empregados, em atividade em Salvador, Bahia. Além disso, buscou-se avaliar a participação dos trabalhadores e dos seus representantes no desenvolvimento desses programas e, ao mesmo tempo, definir e aprimorar condutas, procedimentos e instrumentos de inspeção na área de segurança e saúde no trabalho.

Foram utilizados dados primários e secundários. Os dados primários foram coletados pelos próprios autores, dois Auditores Fiscais (médicos do trabalho) lotados na Delegacia Regional do Trabalho na Bahia, no período entre abril e dezembro de 2002, utilizando um questionário (checklist) especificamente elaborado para levantamento dos riscos ambientais presentes nos locais de trabalho. Os dados secundários, por sua vez, foram obtidos valendo-se dos documentos-base, dos históricos e dos relatórios anuais dos PPRA e dos PCMSO elaborados e implementados pelas empresas inspecionadas. Os programas foram analisados e auditorados, tomando como base a identificação e o reconhecimento dos riscos ambientais, assim como a avaliação do cumprimento das etapas, das ações e das metas previstas em seus documentos-base e respectivos relatórios anu- ais. As análises incluíram, ainda, as estratégias e as metodologias utilizadas pelas empresas para o desenvolvimento de seus respectivos programas.

\section{Aspectos conceituais}

Para efeito deste trabalho, foram considerados "riscos ambientais", os agentes químicos, físicos e biológicos existentes nos ambientes de trabalho que, em função de sua natureza, concentração ou intensidade e tempo de exposição, são capazes de causar danos à saúde do trabalhador, conforme conceituação adotada pela NR-9, item 9.1.5, da Portaria 3.214/78 1.

$\mathrm{Na}$ análise do PPRA, considerou-se "inconsistência”, a qualidade ou estado de falta de consistência, de fundamento ou de coerência entre os dados e informações contidas nos documentos fornecidos pela empresa e aqueles verificados fisicamente no local de trabalho pelos Auditores Fiscais do Trabalho. Neste sentido, foram avaliadas as seguintes etapas do PPRA: reconhecimento dos riscos ambientais, estabelecimento de prioridades e metas de avaliação e controle, avaliação quantitativa dos riscos e da exposição dos trabalhadores e, implantação de medidas de controle e avaliação de sua eficácia.

Por sua vez, na análise do PCMSO, considerou-se "inconsistência", a qualidade ou estado de falta de consistência, de fundamento ou de coerência entre os riscos à saúde dos trabalhadores e os procedimentos médicos efetivamente realizados no desenvolvimento do programa médico. Assim, avaliaram-se as seguintes etapas: identificação dos riscos ocupacionais a que está ou será exposto cada trabalhador da empresa, realização dos exames médicos ocupacionais, execução e interpretação dos indicadores biológicos, planejamento anual das ações de saúde, registro dos dados em prontuário clínico individual e emissão do Atestado de Saúde Ocupacional (ASO).

\section{A amostra}

Foram selecionadas trinta empresas. Dez delas de cada um dos setores da indústria, comércio e serviços. A escolha foi realizada por sorteio aleatório, com base em consulta parametrizada no banco de dados do Sistema Federal de Inspeção do Trabalho (Ministério do Trabalho). Os parâmetros utilizados foram empresas com mais de cem empregados em atividade na cidade de Salvador, durante o ano de 2002. Cumpre observar que essa amostra representa $6,5 \%$ das empresas com mais de cem 
empregados, em atividade na citada região, nos referidos setores. Em Salvador, durante o ano de 2002, nos setores da indústria, comércio e serviços, estavam em atividade $465 \mathrm{em}$ presas com mais de cem empregados.

\section{Empresas inspecionadas}

Das 30 empresas inspecionadas, $10(33,3 \%)$ delas pertencem ao setor Industrial, 10 (33,3\%) ao setor de Serviços e 10 (33,3\%) ao setor de Comércio.

Em relação ao grau de risco da atividade econômica desenvolvida, 11 (36,7\%) das empresas foram classificadas como grau de risco dois, $17(56,7 \%)$ como grau de risco três e 2 $(6,7 \%)$ como grau de risco quatro, de acordo com o Quadro I da NR-4 da Portaria 3214/78 1.

Quanto ao número de empregados, todas as empresas selecionadas mantêm mais de 100 trabalhadores em atividade: $15(50,0 \%)$ delas possuem entre 100 e 200 empregados; 4 (13,3\%) entre 201 e 300 empregados; 4 (13,3\%) entre 301 e 400 empregados; $2(6,7 \%)$ entre 401 e 500 empregados; e $5(16,7 \%)$ possuem mais de 500 empregados.

\section{Resultados}

\section{PPRA: resultado das auditorias}

Os PPRA foram analisados, tomando como base a identificação e o reconhecimento dos riscos ambientais existentes nos locais de trabalho, assim como a avaliação do cumprimento das etapas, das ações e das metas previstas em seus documentos-base e respectivos relatórios anuais. Os resultados são apresentados na Tabela 1, tendo sido analisados os seguintes tópicos:

\section{a) Elaboração do programa}

Todas as empresas, independentemente do número de empregados ou do grau de risco de suas atividades, estão obrigadas a elaborar e implementar o PPRA, de acordo com a NR-9, item 9.1.1, da Portaria 3214/78. Das trinta empresas inspecionadas, $2(6,7 \%)$ não tinham elaborado e implementado o referido programa.

\section{b) Responsabilidade técnica}

De acordo com a NR-9, item 9.3.1.1, a elaboração e implementação do PPRA poderão ser feitas por qualquer pessoa, ou equipe de pessoas que, a critério do empregador, sejam capazes de desenvolver o disposto na norma. En- tre as 28 empresas inspecionadas que elaboraram o PPRA, em 14 (50,0\%) delas o programa tinha sido elaborado por um engenheiro de segurança do trabalho, em $13(46,4 \%)$ por um técnico de segurança do trabalho e em 1 (3,6\%) por um médico do trabalho. Embora seja avaliado essencialmente como um programa de higiene ocupacional, em nenhuma das empresas o programa tinha sido elaborado por um profissional higienista ocupacional.

\section{c) Avaliação anual}

A norma estabelece que a empresa deve adotar mecanismos de avaliação que permitam verificar o cumprimento das etapas, das ações e das metas previstas. De acordo com a NR-9, item 9.2.1.1, uma avaliação global do PPRA deve ser efetuada, sempre que necessário e pelo menos uma vez ao ano, para a avaliação do seu desenvolvimento e realização dos ajustes necessários e estabelecimento de novas metas e prioridades. Entre as 28 empresas inspecionadas que elaboraram o PPRA, 24 (85,7\%) não tinham efetuado pelo menos uma avaliação anual do seu programa.

\section{d) Inconsistências identificadas}

Entre as 28 empresas que elaboraram o PPRA, $26(92,9 \%)$ delas apresentaram algum tipo de inconsistência em seu programa. Analisandose as empresas que apresentaram inconsistências de acordo com as etapas do programa, em $12(42,9 \%)$ delas a inconsistência referia-se ao reconhecimento dos riscos; em $11(39,3 \%)$ diziam respeito à avaliação quantitativa; em 20 $(71,4 \%)$, à implantação de medidas coletivas; em $3(10,7 \%)$, ao planejamento do programa; e em $20(71,4 \%)$ a inconsistência estava relacionada ao cronograma de execução das ações.

Estudando-se as inconsistências conforme os riscos ocupacionais, verificou-se que em $82,1 \%$ dos casos as inconsistências relacionavam-se com os riscos físicos, em $28,6 \%$ essa relação era com os riscos químicos e em nenhum caso as inconsistências estavam relacionadas com os riscos biológicos presentes nos locais de trabalho.

No caso das empresas que apresentaram inconsistências relacionadas com os riscos químicos, em $7,1 \%$ delas a inconsistência referiase ao reconhecimento dos riscos, em 35,7\% à avaliação quantitativa, em 25,0\% à implantação de medidas coletivas e, em $7,1 \%$ à implementação de medidas de proteção individual.

Entre as empresas que apresentaram inconsistências relacionadas com os riscos físi- 
cos, em 35,7\% delas a inconsistência referia-se ao reconhecimento dos riscos, em 35,7\% à avaliação quantitativa, em 60,7\% à implantação de medidas coletivas e, em 17,9\% à implantação de medidas de proteção individual.

$\mathrm{Na}$ análise dos resultados das auditorias de PPRA, estratificados consoante as características das empresas (ramo de atividade, grau de risco e número de empregados), não foi possível evidenciar diferenças estatisticamente significativas.

\section{PCMSO: resultado das auditorias}

Fundamentando-se nos riscos à saúde dos trabalhadores, os PCMSO foram analisados, sobretudo os identificados no PPRA da empresa e aqueles verificados pelos Auditores Fiscais durante a inspeção realizada no local de trabalho. Foi avaliado o cumprimento das ações e das metas previstas no planejamento anual, especialmente no que tange à realização obrigatória dos exames médicos ocupacionais. Os resultados são apresentados na Tabela 2, tendo sido analisados os seguintes tópicos:

\section{a) Elaboração do programa}

Todas as empresas, independentemente do número de empregados ou do grau de risco de suas atividades, estão obrigadas a elaborar e implementar o PCMSO, de acordo com a NR-7, item 7.1.1, da Portaria 3.214/78. Das 30 empresas inspecionadas, $2(6,7 \%)$ não tinham elaborado e implementado o referido programa.

\section{b) Avaliação anual}

A NR-7, em seu item 7.4.6, estabelece que o programa deverá obedecer a um planejamento em que estejam previstas as ações de saúde a serem executadas durante o ano, devendo estas ser objeto de relatório anual. Entre as 28 empresas inspecionadas que elaboraram o PCMSO, 22 (78,6\%) não tinham efetuado uma avaliação anual do seu programa.

\section{c) Inconsistências identificadas}

Entre as 28 empresas que elaboraram o PCMSO, $24(85,7 \%)$ delas apresentaram algum tipo de inconsistência em seu programa. As inconsistências verificadas nos programas relacionavam-se em 50,0\% dos casos com o monitoramento biológico dos riscos físicos, em 7,1\% essa relação era com os riscos químicos e em $7,1 \%$ as inconsistências estavam relacionadas com os riscos biológicos presentes nos locais de trabalho.

No caso das empresas que apresentaram inconsistências em seu PCMSO, em 57,1\% delas a inconsistência relacionava-se à realização dos exames complementares (indicadores biológicos), em $21,4 \%$ à periodicidade dos exames médicos, em 17,9\% à realização dos exames médicos clínicos, em 17,9\% à emissão do atestado de saúde ocupacional e em 3,6\% ao registro de dados em prontuário clínico individual.

Ao analisar as inconsistências, relacionando-as aos vários tipos de exames médicos ocupacionais obrigatórios, verificamos que em

Tabela 1

PPRA: resultados das auditorias. Porcentagem de empresas que realizaram os procedimentos recomendados.

\begin{tabular}{lc}
\hline Procedimentos & $\%$ \\
\hline Elaboração do PPRA & 93,3 \\
Avaliação anual do PPRA & 14,3 \\
Inconsistências no PPRA & 92,9 \\
Inconsistências relacionadas com riscos físicos & 82,1 \\
Inconsistências relacionadas com riscos químicos & 28,6 \\
Inconsistências relacionadas com riscos biológicos & 0,0 \\
Inconsistências no planejamento das ações & 10,7 \\
Inconsistências no reconhecimentos dos riscos ambientais & 42,9 \\
Inconsistências na avaliação quantitativa dos riscos & 39,3 \\
Inconsistências na implementação de medidas coletivas & 71,4 \\
Inconsistências no cronograma de execução das ações & 71,4 \\
\hline
\end{tabular}

Tabela 2

PCMSO: resultados das auditorias. Porcentagem de empresas

que realizaram os procedimentos recomendados.

\begin{tabular}{lr}
\hline Procedimentos & $\%$ \\
\hline Elaboração do PCMSO & 93,3 \\
Avaliação anual do PCMSO & 21,4 \\
Inconsistências no PCMSO & 85,7 \\
Inconsistências relacionadas com riscos físicos & 50,0 \\
Inconsistências relacionadas com riscos químicos & 7,1 \\
Inconsistências relacionadas com riscos biológicos & 7,1 \\
Inconsistências relacionadas com o prontuário clínico individual & 3,6 \\
Inconsistências relacionadas com o atestado de saúde ocupacional & 17,9 \\
Inconsistências na realização dos exames médicos clínicos & 17,9 \\
Inconsistências na periodicidade dos exames médicos ocupacionais & 21,4 \\
Inconsistências na realização dos exames médicos complementares & 57,1 \\
\hline
\end{tabular}


$71,4 \%$ das empresas a inconsistência verificada no PCMSO estava relacionada com o exame periódico, em 3,6\% com o exame admissional e, em $3,6 \%$, com o exame médico demissional.

$\mathrm{Na}$ análise dos resultados das auditorias de PCMSO, estratificados de acordo com as características das empresas (ramo de atividade, grau de risco e número de empregados), não foi possível evidenciar diferenças estatisticamente significativas.

\section{O mapa de riscos}

O mapa de riscos, previsto na NR-5 da Portaria $3214 / 781$, tem por objetivo reunir as informações necessárias para estabelecer o diagnóstico da situação de segurança e saúde no trabalho na empresa. Elaborar o mapa de riscos é atribuição dos integrantes da Comissão Interna de Prevenção de Acidentes (CIPA), com a participação do maior número de trabalhadores. Na elaboração do mapa, busca-se conhecer o processo de trabalho, identificar os riscos existentes no local analisado e, ao mesmo tempo, possibilitar a troca e divulgação de informações entre os trabalhadores, bem como estimular sua participação nas atividades de prevenção. O conhecimento e a percepção que os trabalhadores têm do processo de trabalho e dos riscos ambientais presentes, incluindo os dados consignados no mapa de riscos, deverão ser considerados para fins de planejamento e execução do PPRA. Todavia, entre as 30 empresas inspecionadas, $13(43,3 \%)$ não tinham elaborado o mapa de riscos. Entre as 17 empresas que elaboraram o mapa de riscos, em 9 (53,0\%) essa elaboração não tinha contado com qualquer participação dos trabalhadores e em 16 $(94,1 \%)$ não houve a participação de representantes de seus sindicatos, conforme resultados apresentados na Tabela 3 .

\section{Fiscalização estatal: a inspeção trabalhista}

No presente trabalho, analisando as anotações registradas durante os anos de 1995 a 2002 nos Livros de Inspeção do Trabalho (LIT) das empresas estudadas, constatou-se que $8(26,7 \%)$ delas não tinham sido inspecionadas durante o referido período, $3(10,0 \%)$ empresas foram inspecionadas apenas uma vez, $11(36,7 \%)$ foram inspecionadas duas vezes, 3 (10,0\%) foram inspecionadas três vezes, $2(6,7 \%)$ foram inspecionadas quatro vezes e $3(10,0 \%)$ foram inspecionadas cinco ou mais vezes durante o período. Os resultados são apresentados na Tabela 4 .

Após inspecionar as trinta empresas selecionadas, auditorando seus programas PPRA e
PCMSO, foram emitidos 29 Termos de Notificação (TN) para que as empresas regularizassem 215 itens, perfazendo uma média de sete irregularidades por empresa.

\section{Controle social e a participação \\ dos trabalhadores}

A experiência mundial tem demonstrado que a ação da fiscalização estatal é limitada e raramente tem sido suficiente, o que torna cada vez mais importante o controle social, a saber, a fiscalização exercida diretamente pelos próprios trabalhadores e pelos seus sindicatos. De acordo com a NR-9, itens 9.4 e 9.5, os trabalhadores interessados têm o direito de apresentar propostas e receber informações e orientações a fim de assegurar a proteção aos riscos ambientais identificados na execução do PPRA. Além disso, a norma estabelece que colaborar e participar na implantação e na execução do PPRA é uma das responsabilidades dos trabalhadores.

Os documentos-base e os relatórios anuais do PPRA e do PCMSO, por sua vez, devem ser apresentados e discutidos na CIPA. A implantação de medidas de caráter coletivo deve ser sempre acompanhada de treinamento dos trabalhadores, quanto aos procedimentos que assegurem a sua eficiência, e de informação sobre as eventuais limitações de proteção que ofereçam. O trabalhador tem também o direito de receber cópia dos resultados dos procedimentos médicos a que foi submetido, além da segunda via do seu Atestado de Saúde Ocupacional (ASO). Convém destacar, finalmente, que ao estabelecer os parâmetros mínimos e as diretrizes gerais a serem observados na execução dos programas PPRA e PCMSO, as normas estabelecem, também, que os mesmos podem ser ampliados mediante negociação coletiva de trabalho.

No presente estudo, entre as 28 empresas que tinham elaborado o PPRA ou o PCMSO, em nenhuma delas essa elaboração tinha contado com qualquer participação dos trabalhadores (ou de representantes de seu sindicato), e em 25 (89,3\%) os documentos-base do PPRA ou do PCMSO não foram apresentados e discutidos na CIPA. A ampliação das diretrizes gerais e dos parâmetros mínimos dos programas PPRA e PCMSO, por intermédio de negociação coletiva de trabalho, também não foi verificada entre as empresas inspecionadas. Os resultados relacionados com a avaliação do controle social são apresentados na Tabela 5. 


\section{Conclusões}

A legislação brasileira de segurança e saúde no trabalho, a partir do final de 1994, passou a adotar um novo enfoque e estabeleceu a obrigatoriedade das empresas elaborarem e implementarem dois programas: um ambiental, o PPRA, e outro médico, o PCMSO. As novas normas privilegiaram o instrumental clínico-epidemiológico na abordagem da relação saúde/ trabalho e introduziram a questão da valorização da participação dos trabalhadores e do controle social.

No entanto, ao inspecionar trinta empresas baianas com mais de cem empregados, os autores deste estudo evidenciaram que $92,9 \%$ das empresas apresentaram algum tipo de inconsistência em seu programa ambiental (PPRA) e 85,7 \% em seu programa médico (PCMSO).

Após um período de 8 anos de vigência da legislação que introduziu os programas PPRA e PCMSO, os autores constataram que $26,7 \%$ das empresas estudadas não tinham sido inspecionadas pelo menos uma vez, durante o referido período, e que $83,4 \%$ das empresas foram inspecionadas três vezes ou menos, neste período de 1995 a 2002.

Em relação ao controle social, vale dizer, a fiscalização exercida diretamente pelos próprios trabalhadores e pelos seus sindicatos, foi possível comprovar que, entre as empresas que elaboraram o PPRA ou o PCMSO, nenhuma contou com a participação dos trabalhadores ou do sindicato profissional na elaboração. A ampliação das diretrizes gerais e dos parâmetros mínimos dos programas PPRA e PCMSO, por meio de negociação coletiva de trabalho, também não foi verificada entre as empresas inspecionadas.

Concluindo, apesar de as novas normas privilegiarem o instrumental clínico-epidemiológico e valorizarem a participação dos trabalhadores e o controle social, no presente trabalho constataram-se a baixa qualidade técnica dos programas PPRA e PCMSO, a ação limitada e insuficiente da fiscalização estatal dos ambientes de trabalho, além do precário controle social. Os autores apontam a evidente necessidade de ampliar a cobertura da fiscalização estatal, de estimular a participação dos trabalhadores e dos seus representantes no desenvolvimento dos programas PPRA e PCMSO e de desenvolver e aprimorar condutas, procedimentos e instrumentos de inspeção na área de segurança e saúde no trabalho.

Ainda que o espaço para a ação do poder público seja limitado, ele não é pequeno. Além do mais, ganha aceitação nas partes mais de-
Tabela 3

Mapa de Riscos: resultados das auditorias. Porcentagem de empresas que realizaram os procedimentos recomendados.

\begin{tabular}{lr}
\hline Procedimentos & $\%$ \\
\hline Elaboração do Mapa de Riscos & 56,7 \\
Participação dos trabalhadores na elaboração & 47,0 \\
Participação do sindicato dos trabalhadores na elaboração & 5,9 \\
Apresentação do Mapa na reunião da CIPA & 58,8 \\
\hline
\end{tabular}

Tabela 4

PPRA/PCMSO: fiscalização estatal. Porcentagem de empresas

inspecionadas no período de 1995-2002.

\begin{tabular}{lc}
\hline No de inspeções & \% de empresas* \\
\hline Nenhuma & 26,7 \\
1 & 10,0 \\
2 & 36,7 \\
3 & 10,0 \\
4 & 6,7 \\
5 ou mais & 10,0 \\
\hline
\end{tabular}

* Foram estudadas 30 (trinta) empresas

Tabela 5

PPRA/PCMSO: controle social. Porcentagem de empresas

que realizaram os procedimentos recomendados.

\begin{tabular}{lc}
\hline Procedimentos & $\%$ \\
\hline $\begin{array}{l}\text { Participação dos trabalhadores (ou de seus sindicatos) } \\
\text { no desenvolvimento do PPRA ou do PCMSO } \\
\begin{array}{l}\text { Ampliação dos parâmetros do PPRA ou do PCMSO } \\
\text { mediante negociação coletiva de trabalho }\end{array}\end{array}$ & 0,0 \\
$\begin{array}{l}\text { Apresentação do PPRA ou do PCMSO na reunião } \\
\text { da Comissão Interna de Prevenção de Acidentes }\end{array}$ & 10,7 \\
\hline
\end{tabular}

senvolvidas do mundo a idéia do controle social. No âmbito sindical, é forçoso reconhecer que é fundamental uma organização livre e autônoma, mas os sindicatos, por sua vez, têm de se estruturar melhor, assessorarem-se tecnicamente, e principalmente se organizar nos locais de trabalho. Neste sentido, vale destacar algumas propostas que vêm sendo implementadas ao longo dos últimos anos tais como a 
implantação de comissões de saúde nos locais de trabalho, inclusão de cláusulas de segurança e saúde nos acordos e convenções coletivas, criação de departamentos de segurança e saúde nos sindicatos, campanhas educativas, entre outras iniciativas.

Quanto à fiscalização estatal, é clara a necessidade de ampliar rapidamente sua cobertura e eficácia. De início, urge implantar, no serviço público, a prática do planejamento par-

\section{Resumo}

Os autores analisaram Programas de Prevenção de Riscos Ambientais (PPRA) e Programas de Controle Médico de Saúde Ocupacional (PCMSO) implementados por 30 empresas, de diferentes ramos econômicos, com mais de cem empregados, em atividade em Salvador, Bahia. As inconsistências verificadas foram estudadas segundo os riscos ocupacionais, levando-se em conta as diversas etapas de desenvolvimento dos programas. Os Autores constataram a baixa qualidade técnica desses programas e apontam a evidente necessidade de ampliar a cobertura da fiscalização estatal, assim como de estimular a participação dos trabalhadores e dos seus representantes no desenvolvimento dos programas PPRA e PCMSO. Consideram, ainda, de fundamental importância o desenvolvimento e o aprimoramento de condutas, procedimentos e instrumentos de inspeção na área de segurança e saúde no trabalho.

Riscos Ambientais; Saúde Ocupacional; Riscos Ocupacionais

\section{Colaboradores}

Os dois autores tiveram participação igual em todas as fases do trabalho. ticipativo por objetivos tanto das ações como dos programas a serem implementados na área de segurança e saúde no trabalho. Em segundo lugar, cumpre providenciar treinamento adequado e atualização técnica permanente do quadro de inspetores em atividade. Impõemse, ainda, a necessidade da imediata realização de concurso público para contratação de novos auditores fiscais.

\section{Referências}

1. Secretaria de Segurança e Saúde no Trabalho. Legislação de segurança e saúde no trabalho. Brasília: Ministério do Trabalho e Emprego; 1999.

2. Organização Internacional do Trabalho. Conferência Internacional do Trabalho (70ㅇ reunião). Serviços de saúde dos trabalhadores. Informe IV (2). Genebra: Organização Internacional do Trabalho; 1985.

3. Moura MA. Um olhar coletivo sobre o método epidemiológico I. Revista Proteção 1998; maio (77): 40-3.

4. Miranda CR. Inspeção do Trabalho, Epidemiologia e Segurança e Saúde no Trabalho. In: Sindicato Nacional dos Agentes da Inspeção do Trabalho, organizador. A importância da Inspeção do Trabalho. Brasília: Sindicato Nacional dos Agentes da Inspeção do Trabalho; 1999. p. 8-23.

5. Pena PLG. Elementos teóricos e metodológicos para a elaboração do PPRA e do PCMSO. Salvador: Faculdade de Medicina, Universidade Federal da Bahia; 2000.

6. Saad IFSD, Giampaoli E. Programa de Prevenção de Riscos Ambientais - NR-9 Comentada. 4a Ed. São Paulo: Associação Brasileira de Higienistas Ocupacionais; 1999.

7. Secretaria de Segurança e Saúde no Trabalho, Ministério do Trabalho e Emprego. Norma Regulamentadora no 7: nota técnica. Brasília: Secretaria de Segurança e Saúde no Trabalho, Ministério do Trabalho e Emprego; 1996.

8. Dal Rosso S. A Inspeção do Trabalho. Brasília: Sindicato Nacional dos Agentes da Inspeção do Trabalho; 1997.

9. Organização Internacional do Trabalho. Convenção no 81 sobre a inspeção do trabalho. Genebra: Organização Internacional do Trabalho; 1947.

10. Sistema Federal de Inspeção do trabalho. Quadro de auditores fiscais do trabalho. Brasília: Ministério do Trabalho e Emprego; 2002.

11. Fundação Instituto Brasileiro de Geografia e Estatísticas. Cadastro central de empresas. Brasília: Fundação Instituto Brasileiro de Geografia e Estatística; 1999.

Recebido em 9/Jan/2003

Versão final reapresentada em 26/Jun/2003

Aprovado em 21/Out/2003 